\title{
Avaliação da aceitação, crenças, percepção e nível de conhecimento parental acerca da vacina do Papilomavírus Humano
}

\author{
Evaluation of acceptance, beliefs, perception and level of parental knowledge about \\ Human Papillomavirus vaccine
}

Evaluación de aceptación, creencias, percepción y nivel de conocimiento de los padres sobre la vacuna contra el Papilomavirus Humano

André Carneiro de Brito ${ }^{1}$, Wagner do Carmo Costa ${ }^{2}$, Roberto Carlos Cruz Carbonell ${ }^{1}$, Ana lara Costa Ferreira $^{1}$, Leila Braga Ribeiroํ, Fabiana Nakashima1, José Geraldo Ticianeli ${ }^{1}$, Allex Jardim da Fonseca $^{1}$, Luiz Fernando Almeida Machado ${ }^{3}$, Bianca Jorge Sequeira Costa ${ }^{1 *}$.

\section{RESUMO}

Objetivo: Avaliar a aceitação, crenças, e nível de conhecimento dos pais de crianças e adolescentes, entre 9 e 14 anos, acerca da vacina contra o HPV. Métodos: Estudo observacional, descritivo, transversal, quantitativo, envolvendo 300 pais ou responsáveis de crianças e adolescentes entre 9 e 14 anos, realizado entre agosto de 2019 e dezembro de 2020, por meio da aplicação de um questionário semiestruturado. Resultados: Demonstraram associações estatisticamente significativas ser mulher e já ter ouvido falar em HPV $(p=0,003)$, ser mulher e já ter ouvido falar sobre a vacina do HPV $(p=<0,001)$, ter maior nível de escolaridade e vacinar seus filhos contra o HPV $(p=<0,001)$, ter uma religião e acreditar que a vacinação estimularia $o$ início precoce da vida sexual dos filhos $(p=0,01)$ e pertencer às religiões evangélicas e Testemunha de Jeová e não vacinar os filhos contra o HPV $(p=0,01)$. Conclusão: $O$ maior nível de escolaridade dos pais e as crenças religiosas foram primordiais para a adesão à vacinação, ficando clara a necessidade de que os mecanismos de transmissão de informação existentes sejam revistos para que atinjam realmente seus propósitos junto à população de Boa Vista.

Palavras-chave: Vacina, HPV, Pais, Conhecimento, Crenças.

\begin{abstract}
Objective: Evaluate the acceptance, beliefs, and level of knowledge of parents or guardians of children and adolescents, between 9 and 14 years old, about the HPV vaccine. Methods: Observational, descriptive, crosssectional, quantitative study, involving 300 parents or guardians of children and adolescents between 9 and 14 years old, carried out between august 2019 and december 2020, through the application of a semistructured questionnaire. Results: Demonstrated statistically significant associations to be female and to have heard of HPV $(p=0.003)$, to be female and to have heard about the HPV vaccine $(p=<0.001)$, to have a higher level of education and to vaccinate their children against HPV $(p=<0.001)$, having a religion and believing that vaccination would stimulate their children's early sexual life $(p=0.01)$ and belonging to the evangelical and Jehovah's Witness religions and not vaccinating children against HPV $(p=0.01)$. Conclusion: For the studied sample, the higher level of education of parents and religious beliefs were essential for adherence to vaccination, making it clear the need for the existing information transmission mechanisms to be revised so that they really reach their goals. purposes with the population of Boa Vista.
\end{abstract}

Key words: Vaccine, HPV, Parents, Knowledge, Beliefs.

\section{RESUMEN}

Objetivo: Evaluar la aceptación, creencias y nivel de conocimiento de los padres de niños y adolescentes, entre 9 y 14 años, sobre la vacuna contra el VPH. Métodos: Estudio observacional, descriptivo, transversal, cuantitativo, que involucró a 300 padres de niños y adolescentes entre 9 y 14 años, realizado entre agosto de 2019 y diciembre de 2020, mediante la aplicación de un cuestionario semiestructurado. Resultados: Demostraron asociaciones estadísticamente significativas ser mujer y haber oído hablar del VPH $(p=0,003)$, ser mujer y haber oído hablar de la vacuna contra el VPH $(p=<0,001)$, tener un mayor nivel de educación y vacunar a sus hijos contra el VPH $(p=<0,001)$, tener religión y creer que la vacunación estimularía la iniciación

\footnotetext{
${ }^{1}$ Universidade Federal de Roraima (UFRR), Boa Vista - RR. *E-mail: bianca.costa@ufrr.br

2 Assembleia Legislativa de Roraima (ALE-RR), Boa Vista - RR.

3 Universidade Federal do Pará, Belém - PA.
}

SUBMETIDO EM: 2/2021

PUBLICADO EM: 3/2021 
sexual temprana de los niños $(p=0,01)$ y pertenecer a las religiones evangélica y Testigo de Jehová y no vacunar a los niños contra el VPH $(p=0,01)$. Conclusión: El mayor nivel de educación de los padres y creencias religiosas fueron fundamentales para la adherencia a la vacunación, dejando clara la necesidad de revisar los mecanismos de transmisión de información existentes para que realmente logren sus propósitos con la población de Boa Vista.

Palabras clave: Vacuna, VPH, Padres, Conocimiento, Creencias.

\section{INTRODUÇÃO}

O Papilomavírus humano (HPV) é um vírus constituído por Ácido desoxirribonucleico (DNA) (NIKOLAKIS G, et al., 2015; EGAWA N, et al., 2017). A infecção por esse vírus é considerada a infecção sexualmente transmissível (IST) mais prevalente no mundo, acometendo a maioria das mulheres sexualmente ativas em algum momento de suas vidas, sendo o risco de contrair a infecção de 50-80\% ao longo da vida (ZANINIA NV, et al., 2017). Possui diversos subtipos, os quais na maior parte são transientes e assintomáticos, entretanto estima-se que $10 \%$ das mulheres infectadas tornam-se portadoras crônicas, portanto, com risco de desenvolvimento do câncer de colo uterino (WATTS LA, et al., 2009).

Evidências epidemiológicas e laboratoriais apontam que o HPV está associado às ISTs e à carcinogênese do trato genital inferior (PEREIRA RGV, et al., 2016). Ao longo do tempo, foram identificados cerca de 200 subtipos desse vírus, os quais podem ser classificados como de baixo risco oncogênico ou de alto risco oncogênico (EGAWA N, et al., 2017; DAUDT C, et al., 2016; ZARDO GP, et al., 2014). Os subtipos oncogênicos de HPV estão associados a pelo menos seis diferentes tipos de câncer, incluindo o de orofaringe, anal, cervical, vaginal, vulvar e peniano (GRAHAM SV, 2017; IBRAHIM A, et al., 2018; DUNNE EF, et al., 2014). Por sua transmissão mais comum ser por via sexual, o uso de preservativos durante a relação sexual é apontado como uma eficiente ferramenta protetiva. Outra forma de prevenção adotada é a vacinação.

As vacinas são ofertadas pelo Sistema Único de Saúde (SUS), desde 2014, fazendo parte do Calendário Nacional de Vacinação do Adolescente. O público-alvo para vacinação são meninas entre 9 a 14 anos e meninos de 11 a 14 anos de idade incompletos. Portadores do vírus HIV e pessoas que receberam transplante de órgãos, entre 9 e 26 anos de idade, também podem receber a vacina, desde que a mesma, seja prescrita por um médico. Existem duas vacinas contra o HPV disponíveis no mundo, a quadrivalente, que protege contra os subtipos $6,11,16$ e 18 e a bivalente, que confere proteção contra os subtipos 16 e 18 (BRASIL, 2014). No Brasil, é disponibilizada a vacina do tipo quadrivalente.

Essa vacina impede a infecção, pelos tipos de HPV nela contidos, quando tomada por quem nunca teve contato com o vírus anteriormente (DAUDT C, et al., 2016). A vacina é realizada em esquema duplo sendo fornecida pelo Sistema Único de Saúde (SUS), por meio de um programa de vacinação específico (BRASIL, 2014). Entretanto, apesar da disponibilização da vacina contra o HPV, a taxa de vacinação no Brasil ainda está aquém do esperado, fato que pode ser influenciado por diversos fatores culturais e sociodemográficos. A adesão à vacinação está diretamente associada aos responsáveis legais, uma vez que na maioria das vezes são eles que são informados sobre o calendário vacinal e que se responsabilizam por levar seus filhos até às Unidades Básicas de Saúde para serem vacinados.

Uma das dificuldades relacionadas a baixa cobertura vacinal contra o HPV é a falta de conhecimento dos pais de crianças e adolescentes acerca da eficácia e segurança da vacina, contudo medidas educacionais abrangendo essa população auxiliam a tomada de decisão para a vacinação (SOUSA PDL, et al., 2018). Desta forma, com base no cenário descrito, este estudo objetiva avaliar a aceitação, crenças, percepção e nível de conhecimento dos pais ou responsáveis de crianças e adolescentes, entre 9 e 14 anos, acerca da vacina contra o HPV em Boa Vista, Roraima, nos anos de 2019 e 2020.

\section{MÉTODOS}

Trata-se de um estudo observacional, descritivo, transversal, quantitativo, envolvendo 300 participantes, pais ou responsáveis de crianças e adolescentes entre 9 e 14 anos. Foi realizado entre agosto de 2019 e dezembro de 2020, por meio da aplicação de um questionário semiestruturado, contendo questões que visavam o levantamento de dados sociodemográficos e de informações sobre o conhecimento, crenças e 
percepções acerca da vacina contra o HPV. Foram incluídos no estudo indivíduos de ambos os sexos, maiores de 18 anos, brasileiros, pais ou responsáveis de crianças e adolescentes entre 9 e 14 anos e que assinaram o Termo de Consentimento Livre e Esclarecido (TCLE). O recrutamento dos participantes ocorreu em 03 Unidades Básicas de Saúde de Boa Vista, capital de Roraima.

Os dados coletados foram tabulados no software Microsoft Excel versão 10. Foram realizadas análises descritivas e inferenciais. Utilizou-se o programa Epi Info (CDC) versão 7.2.3.1 para a análise univariada, feita por meio do teste do $x^{2}$, considerando o nível de significância de $5 \%(p<0,05)$. Este estudo foi aprovado pelo Comitê de Ética em Pesquisa da Universidade Federal de Roraima sob o Parecer n 3.041.025.

\section{RESULTADOS}

As características sociodemográficas dos 300 participantes do estudo podem ser visualizadas na Tabela 1.

Tabela 1 - Características sociodemográficas dos participantes do estudo.

\begin{tabular}{cccc}
\hline Variável & & Freq. Absoluta (n) & Freq. Relativa (\%) \\
\hline \multirow{2}{*}{ Sexo } & Masculino & 153 & 51,0 \\
& Feminino & 147 & 49,0 \\
\hline \multirow{3}{*}{ Faixa Etária } & $21-30$ anos & 44 & 14,7 \\
& $31-40$ anos & 179 & 59,7 \\
& $41-50$ anos & 54 & 18,0 \\
\hline \multirow{3}{*}{ Renda Familiar } & $51-60$ anos & 23 & 19,7 \\
Mensal & Até 1 salário & 59 & 61,3 \\
& Entre 1 e 3 salários & 184 & 18,0 \\
& Entre 4 e 5 salários & 54 & 1,0 \\
\hline & Acima de 5 salários & 3 & 0,6 \\
& Fundamental incompleto & 2 & 7,3 \\
& Fundamental completo & 22 & 4,0 \\
& Médio incompleto & 12 & 35,7 \\
& Médio completo & 107 & 1,7 \\
& Superior incompleto & 5 & 40,0 \\
& Superior completo & 120 & 10,7 \\
\hline \multirow{2}{*}{ Possui religião } & Pós-graduação & 32 & 57,0 \\
& Sim & 171 & 43,0 \\
\hline & Não & 129 & 0,6 \\
& Budista & 1 & 0,6 \\
Religião & Candomblecista & 1 & 37,4 \\
& Católica & 64 & 19,9 \\
& Espírita & 34 & 33,9 \\
& Evangélica & 58 & 7,6 \\
\hline
\end{tabular}

Fonte: Brito AC, et al., 2021.

Sobre o conhecimento dos participantes acerca do HPV, 218 (72,7\%) afirmaram ter ouvido falar no tema. Entretanto, quando questionados sobre o que o HPV poderia causar, $112(37,3 \%)$ não souberam responder. Das 147 mulheres, $118(80,3 \%)$ já ouviram falar sobre HPV, enquanto dentre os 153 homens essa frequência foi $65,4 \%$. As afirmações mais frequentes foram que o HPV poderia causar uma IST $(32 / 10,7 \%)$, câncer (29/9,7\%) e verrugas (29/9,7\%). Na amostra avaliada, 224 indivíduos (74,7\%) afirmaram já ter ouvido falar na vacina contra o HPV. Quando questionados se a vacina preveniria o câncer de colo de útero, $85 \%$ relatou acreditar que sim.

Todavia, 53 participantes (17,7\%) afirmaram que a vacina estimularia o início precoce da vida sexual dos seus filhos. Sobre a relação entre o gênero e o fato de ter ouvido falar na vacina, 124 mulheres $(84,4 \%)$ e 153 homens $(65,4 \%)$ relataram já ter ouvido falar. Dos 53 participantes que acreditavam que a vacinação contra o HPV estimularia o início precoce da vida sexual dos seus filhos, 17 (32,1\%) tinham essa crença por questões religiosas, $17(32,1 \%)$ porque despertaria a curiosidade sobre sexualidade, $06(11,3 \%)$ porque afirmaram que 
os filhos já estão muito despertos para a sexualidade e que a vacina seria um incentivo extra, $4(7,6 \%)$ porque se sentiriam obrigados a conversar com os filhos sobre sexualidade a partir da vacinação, $2(3,8 \%)$ disseram não saber o motivo e $7(13,1 \%)$ não responderam.

Ao avaliar se o fato de possuir ou não uma religião influenciava na crença de que a vacinação contra o HPV estimularia o início precoce da vida sexual dos seus filhos, observou-se que $20,5 \%$ das pessoas que tinham religião e $14 \%$ das que não possuíam relataram possuir esta crença. Quando os participantes que possuíam religião, foram questionados se acreditavam que a vacinação estimularia o início precoce da vida sexual dos filhos, $4(6,25 \%)$ católicos afirmaram que sim, 3 espíritas (8,8\%), 20 evangélicos $(34,5 \%)$ e 08 Testemunhas de Jeová $(61,5 \%)$.

Quanto à relação entre a escolaridade e a crença de que a vacinação contra o HPV estimularia o início precoce da vida sexual dos seus filhos, tem-se que dentre os 267 que possuíam mais anos de estudo (a partir do ensino médio completo) 16,5\% afirmou que sim, enquanto entre os $33 \mathrm{com}$ menos anos de estudo (entre ensino fundamental incompleto e médio incompleto) esse resultado foi de $27,3 \%$. De acordo com a Tabela 2, observou-se que 165 participantes (55\%) não vacinaram seus filhos e que dentre os que vacinaram $14,8 \%$ afirmou que o filho tomou apenas uma dose da vacina. O principal motivo apontado pelos participantes para a vacinação foi prevenção $(35 / 25,9 \%)$, enquanto para a não vacinação foi falta de tempo $(21 / 12,7 \%)$ e falta de informação $(18 / 10,9 \%)$.

Tabela 2 - Conduta dos pais quanto à vacinação contra o HPV.

\begin{tabular}{|c|c|c|c|}
\hline Questão & & $\begin{array}{l}\text { Frequência } \\
\text { absoluta }(n)\end{array}$ & $\begin{array}{l}\text { Frequência } \\
\text { relativa (\%) }\end{array}$ \\
\hline $\begin{array}{c}\text { Seu filho (a) foi vacinado (a) } \\
\text { contra o HPV? }\end{array}$ & $\begin{array}{l}\text { Sim } \\
\text { Não }\end{array}$ & $\begin{array}{l}135 \\
165 \\
\end{array}$ & $\begin{array}{l}45,0 \\
55,0 \\
\end{array}$ \\
\hline $\begin{array}{c}\text { Caso seu filho (a) tenha sido } \\
\text { vacinado (a), ele (a) tomou as } \\
\text { duas doses? }\end{array}$ & $\begin{array}{c}\text { Sim } \\
\text { Não } \\
\text { Sem resposta }\end{array}$ & $\begin{array}{c}114 \\
20 \\
1\end{array}$ & $\begin{array}{c}84,0 \\
14,8 \\
0,8\end{array}$ \\
\hline $\begin{array}{l}\text { Você tem ou teve algum receio } \\
\text { em vacinar seu filho (a) contra o } \\
\text { HPV? }\end{array}$ & $\begin{array}{l}\text { Sim } \\
\text { Não }\end{array}$ & $\begin{array}{c}34 \\
266\end{array}$ & $\begin{array}{l}11,3 \\
88,7\end{array}$ \\
\hline $\begin{array}{l}\text { Caso seu filho (a) tenha sido } \\
\text { vacinado (a), qual o motivo que } \\
\text { lhe levou a vaciná-lo (a)? }\end{array}$ & $\begin{array}{c}\text { Combate ao HPV } \\
\text { Cuidado } \\
\text { Evitar adoecimento } \\
\text { Evitar câncer } \\
\text { Evitar câncer de colo de útero } \\
\text { Informação } \\
\text { Outra filha já teve HPV } \\
\text { Prevenção } \\
\text { Proteção } \\
\text { Sem resposta }\end{array}$ & $\begin{array}{c}2 \\
21 \\
9 \\
5 \\
3 \\
2 \\
1 \\
35 \\
12 \\
45\end{array}$ & $\begin{array}{c}1,6 \\
15,5 \\
6,7 \\
3,7 \\
2,2 \\
1,6 \\
0,7 \\
25,9 \\
8,8 \\
33,3\end{array}$ \\
\hline $\begin{array}{l}\text { Caso seu filho (a) não tenha sido } \\
\text { vacinado (a), qual o motivo que } \\
\text { Ihe levou a não vaciná-lo (a)? }\end{array}$ & $\begin{array}{c}\text { Ainda vacinarei } \\
\text { A criança não quis ser vacinada } \\
\text { Criança não pega essa doença } \\
\text { Estimulará a prática sexual precoce } \\
\text { Essa infecção só ocorre em meninas } \\
\text { É pecado } \\
\text { Falha minha } \\
\text { Falta de tempo } \\
\text { Falta de informação } \\
\text { Já vacinei contra a gripe } \\
\text { Medo de vacinas } \\
\text { Meu filho (a) é muito novo (a) } \\
\text { Vacina faltando na UBS } \\
\text { Não sei }\end{array}$ & $\begin{array}{c}7 \\
5 \\
7 \\
12 \\
14 \\
4 \\
14 \\
21 \\
18 \\
1 \\
2 \\
12 \\
2 \\
46 \\
\end{array}$ & $\begin{array}{c}4,2 \\
3,0 \\
4,2 \\
7,4 \\
8,5 \\
2,4 \\
8,5 \\
12,7 \\
10,9 \\
0,6 \\
1,2 \\
7,3 \\
1,2 \\
27,9\end{array}$ \\
\hline
\end{tabular}

Fonte: Brito AC, et al., 2021 
Dos 267 participantes com mais anos de estudo, 131 (49\%) vacinaram seus filhos, enquanto entre os 33 com menos anos de estudo esse resultado foi de $12,1 \%$. A relação entre a religião do participante e 0 ato de vacinar o filho é apresentada na Figura 1.

Figura 1 - Relação entre a vacinação dos filhos e a religião dos participantes do estudo.

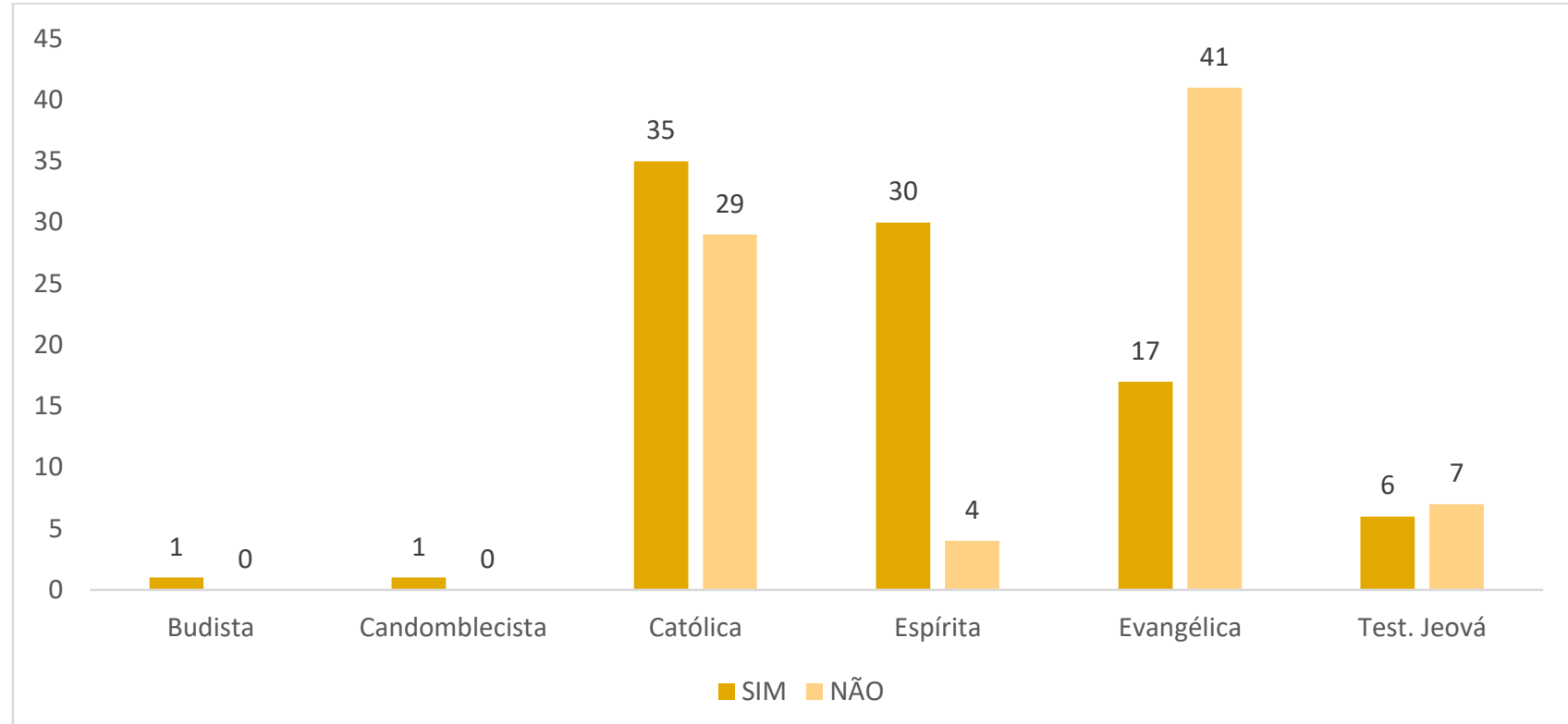

Fonte: Brito AC, et al., 2021

Quando questionados se conversavam sobre sexualidade com seus filhos, 92 mulheres (63\%) e 89 $(58,6 \%)$ homens afirmaram que sim (Figura 2).

Figura 2 - Relação entre o gênero dos participantes do estudo e o ato de conversar sobre sexualidade com seus filhos.

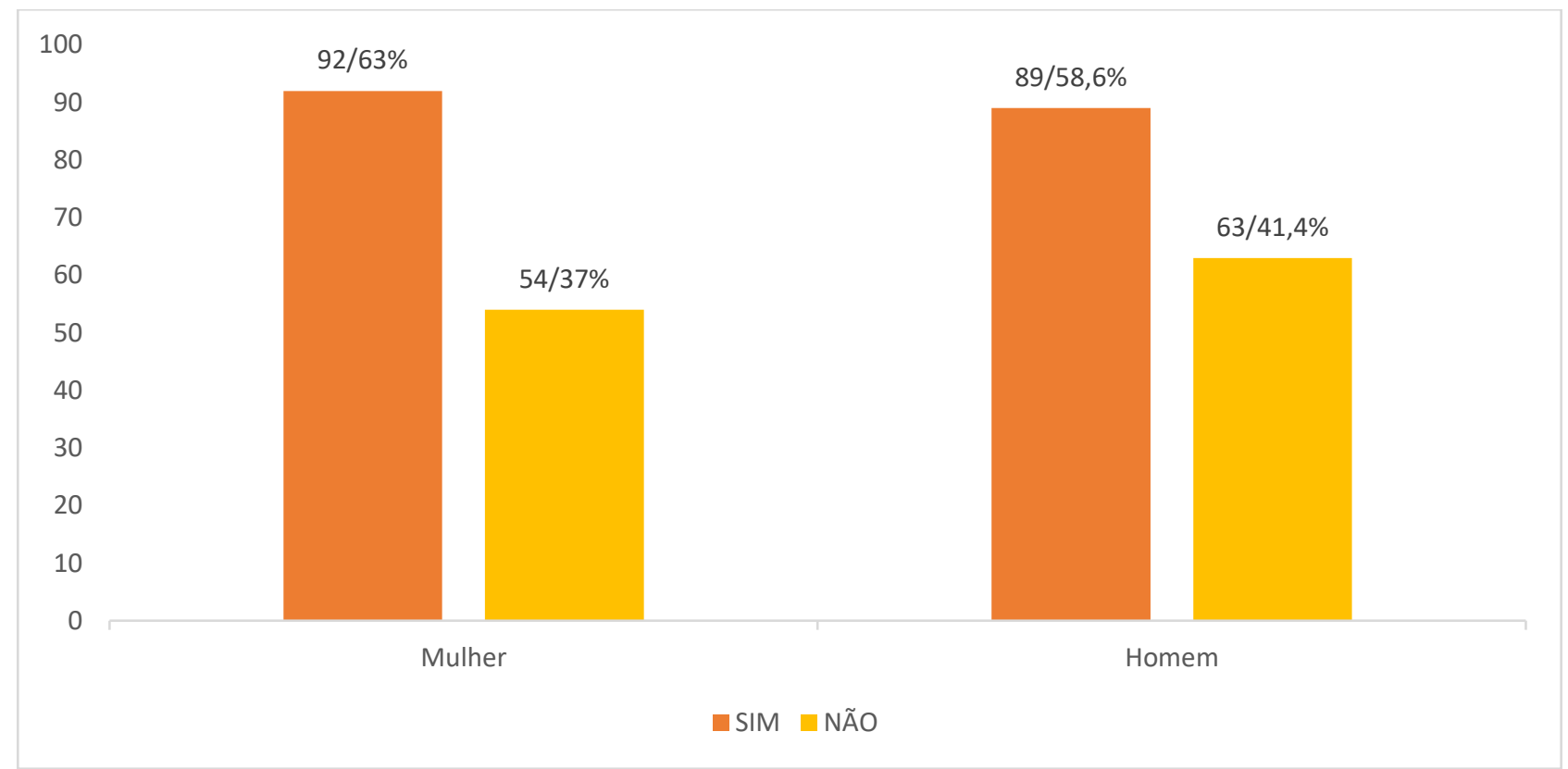

Fonte: Brito AC, et al., 2021

Das 171 pessoas que afirmaram possuir uma religião, 107 (62,9\%) conversavam sobre sexualidade com os filhos e $63(37,1 \%)$ não. Por outro lado, entre as 129 que não tinham religião, 74 (57,8\%) conversavam e $54(42,2 \%)$ não. Evidenciou-se ainda que 41 de 64 católicos conversavam com os filhos sobre sexualidade, 
seguidos de 32 dos 34 espíritas, de 26 dos 58 evangélicos e de 7 dos 13 Testemunhas de Jeová. Já, no tocante ao nível de escolaridade e a ocorrência de conversas sobre sexualidade, observou-se que dentre os 267 com maior escolaridade, $64,8 \%$ afirmou conversar com os filhos, enquanto dentre os 33 participantes com menos anos de estudo esse resultado foi de $25,8 \%$.

Por meio do teste do qui-quadrado, foram evidenciadas as seguintes a associações estatisticamente significativas: ser do gênero feminino e já ter ouvido falar em $\operatorname{HPV}(p=0,003)$, ser do gênero feminino e já ter ouvido falar sobre a vacina do HPV $(p=<0,001)$, ter maior nível de escolaridade e vacinar seus filhos contra 0 HPV ( $p=<0,001)$, ter maior nível de escolaridade e conversar com os filhos sobre sexualidade $(p=<0,001)$.

Foi evidenciada ainda associação significativa entre ter uma religião e acreditar que a vacinação estimularia $o$ início precoce da vida sexual dos filhos $(p=0,01)$, pertencer às religiões evangélicas e Testemunha de Jeová e não vacinar os filhos contra o HPV $(p=0,01)$, ser evangélico ou Testemunha de Jeová e não conversar sobre sexualidade com os filhos $(p=<0,001)$, bem como ser evangélico ou Testemunha de Jeová e acreditar que a vacinação estimularia o início precoce da vida sexual dos filhos $(p=<0,01)$. Além disto, foi observado que ser espírita está associado a uma maior adesão à vacinação dos filhos contra o HPV $(p=<0,01)$.

\section{DISCUSSÃO}

Neste estudo, a maioria dos participantes (51\%) é do sexo masculino e possui um nível mais alto de escolaridade, ou seja, a partir do ensino médio completo $(50,7 \%)$, divergindo de pesquisas que apontam participação irrisória de homens e um nível de escolaridade inferior ao ensino médio completo entre os participantes (SAQER A, et al., 2017; SILVA TIM, et al., 2017; ZANINIA NV, et al., 2017). Com relação à renda familiar mensal, $61,3 \%$ dos participantes recebiam entre 1 e 3 salários-mínimos, resultado corroborado por outros estudos (SAQER A, et al., 2017; SILVA TIM, et al., 2017). O mesmo comportamento foi evidenciado quanto à religião, uma vez que este estudo corrobora com outros, ao apontar que a maioria dos participantes eram católicos (37,4\%) (SILVA TIM, et al., 2017; ZANINIA NV, et al., 2017).

Sobre o conhecimento acerca do HPV, 72,7\% afirmou já ter ouvido falar sobre o vírus. Entretanto, quando questionados sobre o que ele poderia causar, $112(37,3 \%)$ não souberam responder, ou seja, o conhecimento sobre o tema ainda é bastante superficial, fato que pode estar influenciando na baixa cobertura vacinal em Roraima. Outros estudos apontam que o conhecimento ainda é menor entre homens, pessoas que utilizam o serviço de saúde pública e com baixa escolaridade (PIMENTA ATM, et al., 2014; ABREU MNS, et al., 2018; OSIS MJD, et al., 2014).

Observa-se que $80,3 \%$ das mulheres e $65,4 \%$ dos homens já ouviu falar sobre o vírus. Esta distinção pode ser evidenciada em vários outros estudos, indicando que as mulheres possuem maior intimidade com o tema, pelo fato de que por muito tempo o HPV foi associado apenas ao câncer de colo uterino, sem ser mencionado que também poderia acometer os homens (ABREU MNS, et al., 2018; NOSAYABA-OSAZUWA P, et al., 2017). Mulheres têm 1,69 vezes mais chance de conhecer sobre o HPV que homens (ABREU MNS, et al., 2018).

Quando questionados sobre o que a infecção pelo HPV poderia causar, as respostas corretas foram: IST (32/10,7\%), câncer (29/9,7\%) e verrugas (29/9,7\%). Percebe-se que, apesar da maior parte dos participantes terem um nível de escolaridade mais alto, isto não é proporcional ao conhecimento sobre o HPV, uma vez que apenas 30\% responderam corretamente. De acordo com Pereira RGV, et al. (2016), tal fato é preocupante, uma vez que o conhecimento superficial ou incorreto sobre o HPV, subestima sua relação com a saúde da população, influenciando na busca pelos mecanismos de sua prevenção. Os cuidados com a saúde tornam-se piores na medida em que cresce o desconhecimento das ferramentas para este.

Diferente dos resultados deste estudo, outras pesquisas relatam maior percentual de participantes que associaram o HPV corretamente ao câncer de colo uterino ou ao aparecimento de verrugas (63,5\%), (48\%), (52\%) (PIMENTA ATM, et al., 2014; ZANINIA NV, et al., 2017; PEREIRA RGV, et al., 2016).

Talvez, essa diferença possa ser explicada por questões socioculturais ou por uma falha no processo de disseminação de informação. Como principais fontes de informação sobre o HPV ou a vacina, os participantes apontaram a escola dos filhos (51,8\%), os profissionais de saúde (50\%), a internet (30,7\%), amigos (10\%) e 
rádio e/ou TV (9,2\%). Este resultado demonstra que a escola possui um papel essencial na prevenção das ISTs e que essa ferramenta poderia ser melhor utilizada pelos gestores de saúde. Este resultado diverge de vários outros estudos que apontam as mídias sociais como principais fontes de informações, seguida dos profissionais de saúde (PEREIRA RGV, et al., 2016; SAQER A, et al., 2017; SILVA TIM, et al., 2017).

Sobre a vacina do HPV, $74,7 \%$ dos participantes afirmou que já tinha ouvido falar nela e quando questionados se a vacina preveniria o câncer de colo de útero, $85 \%$ disse que sim. Os achados deste estudo divergem de pesquisas que apontam um percentual inferior a $50 \%$ de pessoas que já tinham ouvido falar na vacina (PEREIRA RGV, et al., 2016; SAQER A, et al., 2017; YU Y, et al., 2016). Grande variação nos índices de cobertura vacinal no Brasil pode ser reflexo do pouco conhecimento acerca do HPV e da sua vacina; por outro lado, países com melhor nível socioeducacional têm a cultura do questionamento, gerando um comportamento mais coerente frente ao assunto (ZANINIA NV, et al., 2017).

Quanto à importância da vacinação, a prevenção da infecção pelo HPV foi a resposta mais citada, embora o quantitativo de participantes que fez esta associação tenha sido pequeno. Este resultado coincide com outros estudos onde a vacina foi muitas vezes relatada como terapêutica e não profilática (SILVA TIM, et al., 2017; SANTOS LB, et al., 2011; PEREIRA RGV, et al., 2016).

Observou-se que 165 (55\%) participantes não vacinaram seus filhos e que dentre os que vacinaram 14,8\% afirmou que o filho tomou apenas uma dose da vacina, ou seja, não concluiu o esquema vacinal. Segundo Silva TIM, et al. (2017), o responsável pelo jovem que irá receber a vacina, pode acreditar, por falta de informação, que com uma única dose a pessoa já fique imunizada não necessitando de outras doses. Esse pensamento faz com que menos jovens compareçam nas unidades de saúde para receber a dose de reforço.

Países menos desenvolvidos, possuem menores taxas de vacinação (GALBRAITH KV, et al., 2016). Preocupações morais e religiosas são comuns nesses países e como muitas religiões pregam a abstinência sexual até o casamento, a vacina torna-se desnecessária na opinião dos religiosos (KRAWCZYK A, et al., 2015).

Nesta pesquisa, os principais motivos alegados para a não vacinação foram: falta de tempo $(12,7 \%)$, falta de informação (10,9\%), crença de que a infecção só acometeria meninas $(8,5 \%)$ e de que a vacinação estimularia o início precoce da vida sexual $(7,4 \%)$. Diversos fatores podem contribuir para a não adesão à vacina contra o HPV, como: conhecimento limitado do HPV, das doenças relacionadas ao HPV, preocupações dos pais em relação a segurança da vacina; além de barreiras religiosas e culturais. Esses fatores impactam diretamente na redução da cobertura vacinal (SAQER A, et al., 2017; RODRIGUES NA, et al., 2017; FARIAS CC, et al., 2016; KHURANAA S, et al., 2015).

Ao alegarem que os filhos homens não precisam ser vacinados porque a infecção pelo HPV só acomete mulheres, os pais, por desinformação, colaboram para uma lacuna na cobertura vacinal e para a não proteção dos filhos, uma vez que a importância da inclusão dos meninos na vacinação contra o HPV é reforçada por estudos que mostram que a inserção dos mesmos na vacinação incrementa a vacinação das meninas, reduz os desfechos relacionados ao HPV e, além disso, previne os cânceres de pênis, ânus, boca, orofaringe e verrugas genitais. Por serem os responsáveis pela transmissão do vírus para suas parceiras, ao receber a vacina colaboram com a redução da incidência do câncer de colo de útero e vulva nas mulheres (BRASIL, 2014; KHURANAA S, et al., 2015).

Fica evidente que a recusa por parte do responsável é uma justificativa frequente para os adolescentes não se vacinarem, destacando a preocupação quanto aos efeitos colaterais, à crença religiosa e a alegação de que a vacina possa promover promiscuidade (ZANINIA NV, et al., 2017). Neste estudo, a crença religiosa demonstrou associação estatisticamente significativa com o ato de não vacinar os filhos, uma vez que foram evidenciadas as seguintes associações: ter uma religião e crer que a vacinação provocaria o início da vida sexual precoce $(p=0,01)$, ser evangélico ou Testemunha de Jeová e não vacinar os filhos $(p=0,01)$, ser evangélico ou Testemunha de Jeová e crer que a vacina estimularia o início precoce da vida sexual $(p<0,001)$ e ainda ser espírita e ter uma maior adesão a vacinação $(p<0,01)$.

Já em países desenvolvidos, onde o nível educacional é maior, a recusa por parte dos pais atua como fator importante na adesão à campanha de vacinação, porém não preponderante, destacando-se, portanto, o medo de efeitos colaterais da vacina contra o HPV. Identificaram-se preocupações mais pragmáticas do que 
as crenças religiosas, como possíveis efeitos colaterais; segurança da vacina; necessidade de maiores informações acerca do HPV, do câncer cervical e da vacina. Tais questionamentos são mais comuns entre moradores de países desenvolvidos, de maior nível socioeconômico e educacional, como a Austrália, os EUA, o Canadá e países europeus, como Holanda, Espanha, Suécia e França (GRANDAHL M, et al., 2014). Tais resultados são diferentes dos apontados no presente estudo.

Como a adolescência é uma fase em que surgem muitas dúvidas, é necessário que os pais compreendam e vivenciem essa fase junto com seus filhos, valorizando conhecimentos, orientando-os e participando ativamente da vida dos mesmos, mostrando que a família é essencial na formação destes indivíduos e contribui na construção de sua identidade (ARAÚJO AVS, et al., 2015; PADILHA AP, et al., PINHEIROS, DN, et al., 2013).

Apesar da comunicação entre pais e filhos ser uma importante ferramenta de proteção contra as ISTs, verificou-se neste estudo que existe uma grande dificuldade para abordar temas que envolvam a sexualidade e que isto também está relacionado às crenças religiosas e ao nível educacional dos pais, uma vez que na amostra estudada, ser evangélico ou Testemunha de Jeová associou-se a não ocorrência de conversas entre pais e filhos $(p<0,001)$ e houve uma correlação entre a maior escolaridade e a realização dessas $(p<0,001)$.

\section{CONCLUSÃO}

Para a amostra estudada, o maior nível de escolaridade dos pais, e algumas crenças religiosas, como o catolicismo e o espiritismo, foram primordiais para a adesão à vacinação. Desta forma, é essencial que os mecanismos de transmissão de informação e de divulgação das campanhas existentes sejam revistos para que atinjam realmente seus propósitos junto a população, dirimindo as principais dúvidas e rompendo os tabus que cercam a vacinação contra o HPV.

\section{AGRADECIMENTOS}

À Secretaria Municipal de Saúde que permitiu a realização do estudo.

\section{REFERÊNCIAS}

1. ABREU MNS, et al. Conhecimento e percepção sobre o HPV na população com mais de 18 anos da cidade de Ipatinga, MG, Brasil. Ciência \& Saúde Coletiva. 2018;23(3):849-860.

2. ARAÚJO AVS, et al. O papel dos pais na educação sexual de adolescentes: uma revisão integrativa. Revista da Universidade Vale do Rio Verde. 2015; 13(2):117- 128.

3. BRASIL. Ministério da Saúde. Secretaria de Vigilância em Saúde. Departamento de Vigilância de Doenças Transmissíveis. Coordenação Geral do Programa de Imunizações. Guia Prático sobre HPV: Perguntas e respostas. Brasília: Ministério da Saúde; 2014. v.1. 198p.

4. DAUDT C, et al. How many papillomavirus species can go undetecdet in papilloma lesions? Scientific Reports. 2016; 6:364-380.

5. DUNNE EF, et al. CDC grand rounds: reducing the burden of HPV-associated cancer and disease. MMWR. 2014; 63(4):69-72.

6. EGAWA N, DOORBAR J. The low-risk papillomaviruses. Elsevier. 2017;231(1):119-127.

7. FARIAS CC, et al. Factors related to non-compliance to HPV vaccination in Roraima - Brazil: A region with a high incidence of cervical cancer. BMC Health Services Research. 2016;16(1):417- 436.

8. GALBRAITH KV, et al. Parental acceptance and uptake of the HPV vaccine among African Americans and Latinos in the United States: A literature review. Social Science \& Medicine. 2016;159: 116-126.

9. GRAHAM SV. The human papillomavirus replication cycle, and its links to cancer progression: a comprehensive review. Clin Sci (Lond). 2017;131(17):2201-2221.

10. GRANDAHL M, et al. Not the right time: why parents refuse to let their daughters have the human papillomavirus vaccination. Acta Pædiatr. 2014;103(4):436-41

11. IBRAHIM A, et al. The importance of high-risk human Papillomavirus types other than 16 and 18 in cervical neoplasia. Archives of Pathology \& Laboratory Medicine. 2018;142(6):693-695.

12. KHURANAA S, et al. HPV vaccine acceptance among adolescent males and their parents in two suburban pediatric practices. Vaccine. 2015;33:1620-1624.

13. KRAWCZYK A, et al. decision-making about the human papillomavirus vaccine for their daughters: II. Qualitative results. Hum Vaccin Immunother. 2015;11(2):330-236.

14. NIKOLAKIS G, et al. Carcinomas de células escamosas induzidas por HPV nos dedos de um paciente com lúpus eritematoso sistêmico: um caso e revisão. Relatos de Casos em Dermatologia. 2015; 7(3):329-334. 
15. NOSAYABA OSAZUWA P, et al. Not just a woman's business! Understanding men and women's knowledge of HPV, the HPV vaccine, and HPV-associated cancers. Preventive Medicine. 2017;99: 299-304.

16. OSIS MJD, et al. Conhecimento e atitude de usuários do SUS sobre o HPV e as vacinas disponíveis no Brasil. Rev Saúde Pública. 2014;48(1):123-133.

17. PADILHA AP, et al. O conhecimento de adolescentes sobre doenças sexualmente transmissíveis. Revista Eletrônica Gestão \& Saúde. 2015; 6(3): 2249-60.

18. PEREIRA RGV, et al. The influence of the knowledge towards the vaccine against Human Papillomavirus: a randomized clinical trial. ABCS Health Sci. 2016;41(2):78-83.

19. PIMENTA ATM, et al. Conhecimento de mulheres sobre alguns aspectos do papilomavírus humano. Medicina (Ribeirão Preto). 2014;47(2):143-8.

20. PINHEIRO DN, et al. Aspectos educativos do programa de prevenção do câncer do colo do útero, Belém, Pará, Brasil. Revista Eletrônica Gestão \& Saúde. 2013;4(4):1469-1482.

21. RODRIGUES AN, et al. An alert to Latin America: Current human papillomavirus vaccination trends highlight key barriers to successful implementation. Cancer. 2017;123(12):2193-2199.

22. SAQER A, et al. Knowledge and awareness about cervical cancer vaccine (HPV) among parents in Sharjah. Asian Pacific Journal of Cancer Prevention. 2017;18:1237-1241.

23. SANTOS LB, et al. Percepção das mães quanto à importância da imunização infantil. Rev Rene. 2011;12(3):621-626.

24. SILVA TIM, et al. Vacina e HPV: saberes dos pais e responsáveis de meninas adolescente. Rev. Gestão \& Saúde (Brasília). 2017;1(03):622-637.

25. SOUSA PDL, et al. Knowledge and Acceptance of HPV Vaccine Among Adolescents, Parents and Health Professionals: construct development for collection and database composition. J Hum Growth Dev. 2018;28(1):58-68.

26. WATTS LA, et al. HPV vaccine: a comparision of attitudes and behavioral perpestives between latino and non-latino women. Gynecol Oncol. 2009;112(3):577-82.

27. YU Y, et al. Human papillomavirus infection and vaccination: Awareness and knowledge of HPV and acceptability of HPV vaccine among mothers of teenage daughters in Weihai, Shandong, China. PLoS One. 2016;11:1-14.

28. ZANINIA NV, et al. Motivos para recusa da vacina contra o Papilomavírus Humano entre adolescentes de 11 a 14 anos no município de Maringá-PR. Rev Bras Med Fam Comunidade. 2017; 2(39):1-13.

29. ZARDO GP, et al. Vacina como agente de imunização contra o HPV. Ciência \& Saúde Coletiva. 2014; 19(9):37993808. 